\title{
8. Observation of the Microcirculatory System in Brain Tumor
}

\author{
Toyozo Aizawa, Tsuneo Hasegawa, Koyu Mori and Keiji Yoshida \\ Department of Internal Medicine, School of Medicine, Keio University \\ Haruo MatsuYama \\ Department of Pathology, School of Medicine, Keio University
}

In 15 autopsied cases, the pattern of the microcirculatory system of Meningioma, Meningeal sarcoma, Astrocytoma, Glioblastoma, Medulloblastoma, Ependymoma, Oligodendroglioma and Hemangioma were observed histologically with Hematoxylin and Eosin preparation, Silver impregnation technique (HasegawaRavens) and Cajal' gold sublimate procedure.

The following results were obtained;

1. The capillaries were extremely irregular, and formed coarse network with abnormal pattern in the brain tumor without fibrosis. On the Oligodendroglioma and Ependymoma, the capillaries were formed regular dense network. And a characteristic relationship between the capillary abnormalities and the malignancy of brain tumor was observed.

2. The proliferation of abnormal vascularities in microcirculatory system was also observed with malignant findings in the tumor and the differentiation between arteriole and venule was somewhat difficult in a few areas of these tumors. Such abnormal vessels in the malignant tumors were diffusely penetrated into normal tissue.

3. The A-V shunts and throughfare channels with abnormality were observed clearly in our cases of Astroblastoma, Glioblastoma and Hemangioma. Some of them were ruptured and hemorrhaged into tumor or peripheral zone.

\section{Diffuse Meningeal Tumor}

\author{
Kazuo TAKeUCHI and Shoji OdA \\ Department of Neurosurgery, Toranomon Hospital, Tokyo
}

Diffuse meningeal tumors are much different from usual intracranial space taking lesions from either pathological or clinical view point.

We have experienced 2 cases of primary and 20 cases of secondary diffuse meningeal tumors since 1958. The former cases were melanoma and sarcoma, both of which are very rare among literatures. The latter cases are meningeal metastases from 12 cases of carcinoma and one case of retroperitoneal lymph- 
sarcoma, and seedings from 7 cases of primary brain tumors. The primary foci of the carcinoma were the stomach ( 7 cases), lung ( 4 cases) and thyroid (one case). Histological classification of the primary brain tumors were as follows: glioblastoma 4 , medulloblastoma 2 , and ependymoma 1 .

The case of melanoma and 3 cases originated from the gastric carcinoma have been diagnosed clinically, but in the remaining cases the diagnosis was first established by autopsy. Because in cases of the diffuse meningeal carcinomatosis from the gastric cancer, signs of meningeal irritation and pleocytosis of CSF were always seen. Generally, pleocytosis of CSF were commonest, but excess of protein in CSF were less common. Discovery of the tumor cells in CSF is very useful for clinical diagnosis.

In most cases intracranial pressure increased markedly because of excessive retention of CSF. Therefore, removal of CSF was most effective symptomatically. From our clinical experiences intrathecal application of radioisotopes or cancerocydal drugs should be studied in the future, though the prognosis of such widespread tumors which thought to be inoperable, is absolutely poor.

\title{
10. A Case of Diffuse Meningeal Melanosis accompanying a Malignant Melanoma in the Region of the Insula
}

\author{
Kazuo Kinoshita, Tooru Soejima and Yoshiaki Takeno \\ Department of Neurosurgery, Neurological Institute, Faculty of Medicine, \\ Kyushu University
}

The patient was an 8-year-old boy who had episodes of generalized convulsive seizures, nausea, vomiting and headache of 3 month's duration.

On admission he had only a papilloedema still in initial stage. An EEG revealed an organic lesion in the left frontal region. A left carotid angiogram demonstrated a quite vascular tumor deep in the left frontal lobe. At operation the left cerebral cortex appeared diffusely black. A dark, brownish, well demarcated tumor mass, melanoma, was removed from the insular region. He recovered well from surgery and was discharged without considerable subsequent deficit after one course of Cobalt radiation therapy. He died 3 months after discharge. No autopsy was done.

\section{Light-microscopic findings:}

In the region of the meningeal melanosis, the subarachnoid space was filled with round, uniform, pigmented cells, which also invaded the perivascular spaces of the brain parenchyma. The melanoma cells showed a considerable pleomorphism 